\title{
Relativistic few-body physics
}

\author{
Wayne Polyzou ${ }^{a b c}$ \\ ${ }^{a}$ The University of Iowa, Department of Physics and Astronomy, Iowa City, IA \\ ${ }^{b}$ This work supported by the US Department of Energy under contract No. DE-FG02- \\ 86ER40286 \\ ${ }^{c}$ For the Proceedings of the 22nd European Few-Body Conference in Kracow Poland
}

\begin{abstract}
I discuss different formulations of the relativistic few-body problem with an emphasis on how they are related. I first discuss the implications of some of the differences with non-relativistic quantum mechanics. Then I point out that the principle of special relativity in quantum mechanics implies that the quantum theory has a Poincaré symmetry, which is realized by a unitary representation of the Poincaré group. This representation can always be decomposed into direct integrals of irreducible representations and the different formulations differ only in how these irreducible representations are realized. I discuss how these representations appear in different formulations of relativistic quantum mechanics and discuss some applications in each of these frameworks.
\end{abstract}

Keywords: Relativistic quantum mechanics, relativistic scattering, Poincaré symmetry, Lorentz invariance

\section{Introduction}

In these proceedings I discuss different formulations of the relativistic few-body problem. The goal is to understand the relation between different formulations of relativistic quantum mechanics that are used in applications. The fundamental assumption underlying all of these formulations is the principle of special relativity in quantum mechanics, which implies that in a box isolated from the rest of the universe it is impossible to use experimental measurements to distinguish different inertial coordinate systems.

In quantum mechanics the experimentally measurable quantities are probabilities, expectation values and ensemble averages. These are preserved if the states and observables identified with different inertial coordinate systems are related by unitary transformations. Wigner [1] showed that this condition is necessary and sufficient for the invariance of these observables with respect to changes of the inertial coordinate system. What he showed is that relativistic invariance in the sense described in the previous paragraph is equivalent to the existence of a unitary representation, $U(\Lambda, a)$, of the Poincaré group (Lorentz + space-time translations). This is common to all relativistic quantum mechanical formulations of the few-body problem. In addition, unitary representations of the Poincaré group can always be decomposed into a direct integrals of irreducible representations. This decomposition is the relativistic analog of diagonalizing the Hamiltonian in non-relativistic quantum mechanics. States in each irreducible subspace transform among themselves via well-defined transformations under the Poincaré group. All of the relevant observables can be computed directly from the irreducible eigenstates.

These irreducible representations are common to all formulations of relativistic few-body quantum mechanics. The equivalence of different models is determined by the equivalence of decompositions into irreducible representations. Different formulations of relativistic quantum mechanics differ how the Hilbert spaces are represented, which can vary from being abstract to concrete. 
We discuss how irreducible representations of the Poincaré group appear in theories that emphasize Lorentz covariance, Poincaré covariance, Euclidean covariance, as well as $2 j+1$ vs. $4 j+2$ component spinors.

\section{Poincaré group}

The Poincaré group has ten infinitesimal generators that satisfy commutation relations characteristic of the Poincaré Lie algebra. They are the Hamiltonian, $H$, the linear momentum, $\mathbf{P}$, the angular momentum $\mathbf{J}$, and the rotationless Lorentz boost generators $\mathbf{K}$. Three of the commutators have the Hamiltonian on the right hand side of the commutation relations

$$
\left[P^{m}, K^{n}\right]=i \delta_{m n} H
$$

Since interactions appear on the right hand side of these commutation relations, between three and ten generators must include interactions 2. This is needed to ensure the consistency of the many possible ways of representing time evolution.

\section{Cluster properties}

The discussion of performing experiments in a box that is isolated from the rest of the universe requires that the unitary representation of the Poincaré group factors into a tensor product of a representation involving the degrees of freedom in the box and another representation involving the remaining degrees of freedom, when the degrees of freedom in the box are isolated from the rest of the universe.

I refer to this property as cluster separability of the unitary representation of the Poincaré group. It means for states describing asymptotically separated subsystems, $s_{1}$ and $s_{2}$, that

$$
U(\Lambda, a) \rightarrow U_{s_{1}}(\Lambda, a) \otimes U_{s_{2}}(\Lambda, a) .
$$

It implies that each generator has a cluster expansion and the generators for each subsystem also must satisfy the Poincaré commutation relations. Because some interactions involve particles in overlapping subsystems, the additional invariance requirements cannot be satisfied without introducing a special class of many-body interactions that are distinguished by being functions of the subsystem interactions. Weinberg [3] suggested that avoiding these difficulties is an important motivation for using local field theory. In spite of the difficulties, Sokolov [4 has solved the problem of constructing the many-body interactions that are needed to restore cluster properties.

If a model satisfies cluster properties in one frame, such as the rest frame, then the invariance of the $S$-matrix means the $S$-matrix will satisfy cluster properties in all frames, even when the unitary representation of the Poincaré group does not. However, when this system is embedded in a larger system, it will not always be in the rest frame of the larger system. In this case the many-body interactions need to be included to restore cluster properties in the rest frame of the larger system.

What happens in a three-body model that satisfies cluster properties in the threebody rest frame, but not at the level of the Poincaré generators is that if one particle is asymptotically separated from an interacting pair of particles in frames other than

the rest frame, the interaction between the pair of particles vanishes. Violations of cluster properties have observable consequences, however these appear to be small for nuclear reactions [5] 


\section{Position in relativistic quantum theory}

Another property of relativistic quantum mechanics is the absence of a consistent position operator. The standard explanation for this is that the energy needed to localize a particle in a sufficiently small spatial region is enough to create pairs of particles and antiparticles. However, one does not need antiparticles understand the problem. If one assumes that a state representing a particle at the origin at time $t=0$ is Lorentz invariant, then properties of the irreducible representations imply that the overlap of states with spacelike separated position do not identically vanish; instead they the fall off exponentially with a scale determined by the particle's Compton wavelength. Formally

$$
\begin{gathered}
\langle m, \mathbf{p} \mid x\rangle=\langle m, \mathbf{p}|U(I, x)| 0\rangle= \\
e^{i p \cdot x}\langle m, \mathbf{p} \mid 0\rangle=e^{i p \cdot x}\langle m, \mathbf{p}|U(\Lambda(p), 0)| 0\rangle= \\
e^{i p \cdot x} \sqrt{\frac{m}{\omega_{m}(\mathbf{p})}}\langle m, \mathbf{0} \mid 0\rangle .
\end{gathered}
$$

This implies

$$
\langle\mathbf{x}, 0 \mid \mathbf{y}, 0\rangle=\int e^{i \mathbf{p} \cdot(\mathbf{x}-\mathbf{y})} \frac{c}{\omega_{m}(\mathbf{p})} d \mathbf{p}=2 \pi i c \frac{K_{1}(m|\mathbf{x}-\mathbf{y}|)}{m|\mathbf{x}-\mathbf{y}|} .
$$

For large $x$ the Bessel function $K_{1}(x)$ decays exponentially in $x$.

One thing that follows from this observation is that the notation of retardation, which is a field observable, it is not an observable for relativistic particles, since they cannot be precisely localized.

\section{Spin}

The treatment of spin seems to distinguish irreducible representations of the Poincaré group from Lorentz covariant theories. Specifically, for spin $1 / 2$ particles, fourcomponent spinors are used in Lorentz covariant theories while spin 1/2 irreducible representations of the Poincaré group involve only two-component spinors. In an experiment measurements of the spin projection can take on only two values.

The relation between these two equivalent treatments of spin can be understood by considering the transformation properties of a Poincaré irreducible basis state

$$
\begin{gathered}
U(\Lambda, 0)|(m, j) \mathbf{p}, \mu\rangle= \\
\sum\left|(m, j) \boldsymbol{\Lambda} p, \mu^{\prime}\right\rangle \sqrt{\frac{\omega_{m}(\boldsymbol{\Lambda} p)}{\omega_{m}(\boldsymbol{p})}} \underbrace{D_{\mu^{\prime} \mu}^{j}\left[B^{-1}(\boldsymbol{\Lambda} p / m) \Lambda B(\mathbf{p} / m)\right]}_{\text {Wigner rotation }} .
\end{gathered}
$$

Because $D_{\mu^{\prime} \mu}^{j}[R]$ is also a $2 j+1$ dimensional representation of $S L(2, \mathbb{C})$ it can be used to decompose the Wigner rotation into a product of finite dimensional representations of Lorentz transformations. Lorentz covariant states can be defined by

$$
|p, j, \sigma\rangle_{c}:=\sum|(m, j) \mathbf{p}, \mu\rangle \sqrt{\omega_{m}(\mathbf{p})} D_{\mu \sigma}^{j}\left[B^{-1}(\mathbf{p} / m)\right] .
$$

With this definition eq. (5) becomes a Lorentz covariant transformation of the covariant states:

$$
U(\Lambda, 0)|p, j, \sigma\rangle_{c}=\sum\left|\Lambda p, j, \sigma^{\prime}\right\rangle_{c} D_{\sigma^{\prime} \sigma}^{j}[\Lambda]
$$

The scalar product of two covariant wave-functions,

$$
\Phi(\mathbf{p}, \sigma):={ }_{c}\langle p, j, \sigma \mid \Phi\rangle,
$$


is

$$
\langle\Psi \mid \Phi\rangle=\bigvee \Psi^{*}(\mathbf{p}, \sigma) \frac{m d \mathbf{p}}{\omega_{m}(\mathbf{p})} D_{\sigma \sigma^{\prime}}^{j}\left[B(\mathbf{p} / m) B^{\dagger}(\mathbf{p} / m)\right] \Phi\left(\mathbf{p}, \sigma^{\prime}\right) .
$$

The new feature is that the Lorentz covariant inner product has a momentum and spin-dependent kernel, which maintains the unitarity of the representation. The problem is that while for $S U(2), U=\sigma_{2} U^{*} \sigma_{2}$, there is no similarity transformation relating representations of $S L(2, C)$ to the complex conjugate representations. Technically this is not needed, because the Wigner functions of the boosts and Lorentz transformation are eventually combined to get a Wigner rotation, where this difference disappears, however the problem is that space reflection relates these inequivalent representations. Since in the representation (9) boosts appear in the kernel of the inner product, the kernel and hence the representation of the Hilbert space will change under space reflection. It is possible to get a Hilbert space representation of space reflection by making the replacement

$$
D_{\sigma \sigma^{\prime}}^{j}\left[B(\mathbf{p} / m) B^{\dagger}(\mathbf{p} / m)\right] \rightarrow D_{\sigma_{1} \sigma_{1}^{\prime}}^{j}\left[B(\mathbf{p} / m) B^{\dagger}(\mathbf{p} / m)\right] \oplus D_{\sigma_{2} \sigma_{2}^{\prime}}^{j}\left[B(-\mathbf{p} / m) B^{\dagger}(-\mathbf{p} / m)\right]
$$

For spin 1/2 this means replacing two-component spinors by four-component spinors. When the Lorentz transformations are combined with the boosts, the Wigner rotations for both representations become identical, recovering the original Poincaré covariant result.

\section{Non-relativistic limits}

In the strong coupling region of QCD there is not enough mathematical control of the theory for precision calculations of scattering reactions. As a consequence, realistic models of the strong interaction are ultimately fit to nucleon-nucleon scattering data. This is certainly the case for the so-called high-precision nuclear potentials. These fits are normally performed by first Lorentz transforming the cross section data from the laboratory frame to the center of momentum frame; and then interactions are adjusted so the cross section in the non-relativistic model reproduces this data with a $\chi^{2}$ per degree of freedom near 1. Relativistic interactions can be computed the same way, replacing the non-relativistic model by a relativistic one. The fits are performed by expressing the cross section in terms of transition matrix elements that are functions the rest-frame momentum of one of the particles.

When this is done, both models will give the same phase shifts as a function of the relative momentum that appears in the scattering amplitude. When this procedure is used there are no relativistic effects in two-nucleon observables. Relativistic effects first appear in the three-body problem, where cluster properties dictate how to embed moving two-body subsystems in the three-particle Hilbert space.

This does not mean that the non-relativistic limit of a relativistic nucleon-nucleon model is small. This distinction is important.

This situation in strong interaction physics is analogous to the one that one would have if the Coulomb potential was replaced by a non-relativistic phenomenological potential that reproduced all fine and hyperfine structure in the Hydrogen atom. In that case there would be no two-body relativistic corrections. The difference with the strong interactions is that there is no method comparable to QED for constructing realistic nucleon-nucleon from QCD.

The important conclusion is that it makes no sense to compute relativistic corrections to a non-relativistic model when the interaction in both models is fit to the same scattering data. 


\section{$7 \quad$ Hilbert space representations}

The most familiar relativistic quantum theory is relativistic quantum field theory. It is instructive relate the description of a free particle in Minkowski quantum field theory, Euclidean quantum field theory and Poincaré invariant quantum mechanics. Different relativistic few-body models are related to one of these representations.

In a free quantum field theory a one-particle state is constructed by smearing the field with a test function of four spacetime variables and applying the resulting operator to the vacuum. The Hilbert space scalar product is

$$
\begin{gathered}
(f, f)=\left\langle 0\left|\phi\left(f^{*}\right) \phi(f)\right| 0\right\rangle=\int f(x)^{*}\langle 0|\phi(x) \phi(y)| 0\rangle f(y) d^{4} x d^{4} y= \\
\int f(x)^{*} \frac{1}{(2 \pi)^{3}} \int d^{4} p \theta\left(p^{0}\right) \delta\left(p^{2}+m^{2}\right) e^{i p \cdot(x-y)} f(y) d^{4} x d^{4} y= \\
\int \tilde{f}^{*}\left(\omega_{m}(\mathbf{p}), \mathbf{p}\right) \frac{d \mathbf{p}}{2 \omega_{m}(\mathbf{p})} \tilde{f}\left(\omega_{m}(\mathbf{p}), \mathbf{p}\right)
\end{gathered}
$$

where

$$
\tilde{f}\left(\omega_{m}(\mathbf{p}), \mathbf{p}\right)=\frac{1}{(2 \pi)^{3 / 2}} \int d \mathbf{x} e^{i \mathbf{p} \cdot \mathbf{x}-i \omega_{m}(\mathbf{p})} g\left(x_{0}, \mathbf{x}\right) .
$$

The corresponding Hilbert space inner product in Euclidean quantum field theory is

$$
\begin{gathered}
(g, g)=\int g\left(-x_{0}, \mathbf{x}\right)^{*} S(x-y) g\left(y_{0}, \mathbf{y}\right) d^{4} x_{e} d^{4} y_{e} \\
\int g\left(-x_{0}, \mathbf{x}\right)^{*} \frac{d^{4} p}{(2 \pi)^{4}} \frac{e^{i p_{e} \cdot(x-y)}}{p^{2}+m^{2}} g\left(y_{0}, \mathbf{y}\right) d^{4} x_{e} d^{4} y_{e} \\
=\int \tilde{g}^{*}\left(x_{0}, \mathbf{p}\right) e^{-\omega_{m}(\mathbf{p}) x_{0}} \frac{d x_{0} d y_{0} d \mathbf{p}}{2 \omega_{m}(\mathbf{p})} e^{-\omega_{m}(\mathbf{p}) y_{0}} \tilde{g}\left(y_{0}, \mathbf{p}\right)
\end{gathered}
$$

where

$$
\tilde{g}\left(x_{0}, \mathbf{p}\right)=\frac{1}{(2 \pi)^{3 / 2}} \int d \mathbf{x} e^{-i \mathbf{p} \cdot \mathbf{x}} g\left(x_{0}, \mathbf{x}\right) .
$$

Both expressions lead to integrals of functions of the three momentum integrated with a Lorentz invariant measure, which is the inner product associated with a mass $m$ irreducible representation of the Poincaré group.

Comparing these expressions implies the following relations between these equivalent representations of a free spinless particle of mass $m$ :

$$
\begin{aligned}
\Psi(\mathbf{p})= & \frac{1}{(2 \pi)^{3 / 2}}\langle\mathbf{p}|\phi(f)| 0\rangle=\int f(\mathbf{x}, t) e^{-i \omega_{m}(\mathbf{p}) t-i \mathbf{p} \cdot \mathbf{x}} \frac{d^{4} x}{\sqrt{2 \omega_{m}(\mathbf{p})}} \\
= & \frac{1}{(2 \pi)^{3 / 2}} \int g\left(\mathbf{x}, x_{0}\right) e^{-\omega_{m}(\mathbf{p}) x_{0}-i \mathbf{p} \cdot \mathbf{x}} \frac{d^{4} x}{\sqrt{2 \omega_{m}(\mathbf{p})}} .
\end{aligned}
$$

These relations determine different realizations of the unitary representations of the Poincaré group. In the Minkowski quantum field theory case the manifestly covariant transformation

$$
f(x) \rightarrow f_{\Lambda, a}(x)=f\left(\Lambda^{-1}(x-a)\right)
$$

is unitary provided the vacuum expectation value of the fields is invariant

$$
W(x-y)=W(\Lambda(x-y))=\langle 0|\phi(x) \phi(y)| 0\rangle .
$$

The relations (15) and the representation (16) lead to explicit expressions for the Poincaré generators for both Euclidean field theory as well as Poincaré irreducible 
quantum theory. The Hamiltonian and boost generators in these two case are found to be

$$
H g(x)=\frac{\partial g}{\partial \tau}(x) \quad K_{i} g(x)=\tau \frac{\partial g}{\partial x_{i}}(x)-x_{i} \frac{\partial g}{\partial \tau}(x)
$$

and

$$
H \Psi(\mathbf{p})=\sqrt{\mathbf{p}^{2}+m^{2}} \Psi(\mathbf{p}) \quad K_{i} \Psi(\mathbf{p})=\frac{i}{2}\left\{H, \frac{\partial}{\partial p_{i}}\right\} \Psi(\mathbf{p}) .
$$

The generators for rotations and translations are the same in all three representations.

The main features to notice are that in both the Euclidean and Minkowski field theory cases the formula for the covariant inner product has a non-trivial kernel. These considerations generalize to interacting theories and the generalization of (15) to interacting theories implies precise relations between the different formulations of relativistic quantum mechanics. The relations between the wave functions imply relations between the corresponding unitary representations of the Poincaré group and their infinitesimal generators

\section{Realizations}

In what follows I discuss different ways of constructing relativistic few-body models. I focus on the structure of the model Hilbert space and representations of the Poincaré group.

\subsection{Covariant constraint dynamics}

In this framework the dynamics is given by solving a set of coupled constraint equations

$$
\begin{aligned}
C_{1} \psi & =\left(p_{1}^{2}+m_{1}^{2}+V_{1}\right) \psi=0 \\
C_{n} \psi & =\left(p_{n}^{2}+m_{n}^{2}+V_{n}\right) \psi=0
\end{aligned}
$$

where integrability requires that constraints commute on solutions (first class constraints)

$$
\left[C_{i}, C_{j}\right] \psi=0
$$

A typical realization is

$$
\left[p_{1}^{2}-p_{2}^{2}, V\right] \psi=0 .
$$

Because the constraints are covariant and satisfy the first class condition they can be used to make a model of a Wightman function, $W(x-y)$ of the form

$$
W=\prod_{i} \delta\left(C_{i}\right)
$$

In this case the inner product is

$$
\begin{gathered}
\langle g \mid f\rangle= \\
\int g^{*}\left(x_{1}, x_{2}\right)\left\langle x_{1}, x_{2}\left|\delta\left(C_{1}\right) \delta\left(C_{2}\right)\right| y_{2}, y_{1}\right\rangle f\left(y_{1}, y_{2}\right) d^{8} x d^{8} y
\end{gathered}
$$

and the transformation

$$
f\left(y_{1}, y_{2}\right) \rightarrow f\left(\Lambda y_{1}+a, \Lambda y_{2}+a\right)
$$

defines a unitary representation for the Poincaré group acting on the two-particle Hilbert space. This method has been used in a number of applications involving both constituent quarks [7] 8 and charged particles [9] [10]. 


\subsection{Direct interaction quantum mechanics}

This formulation of relativistic quantum mechanics is formulated on direct sums of tensor products of single-particle irreducible representation spaces of the Poincaré group. Interactions are added to sums of single-particle generators in a manner that preserves the Poincaré commutation relations.

$$
\begin{gathered}
\mathcal{H}=\oplus\left(\otimes \mathcal{H}_{j m}\right) \\
H=\sum_{i} H_{i}+\sum_{i j} H_{i j}+\sum_{i j k} H_{i j k}+\cdots \\
\mathbf{P}=\sum_{i} \mathbf{P}_{i}+\sum_{i j} \mathbf{P}_{i j}+\sum_{i j k} \mathbf{P}_{i j k}+\cdots \\
\mathbf{J}=\sum_{i} \mathbf{J}_{i}+\sum_{i j} \mathbf{J}_{i j}+\sum_{i j k} \mathbf{J}_{i j k}+\cdots \\
\mathbf{K}=\sum_{i} \mathbf{K}_{i}+\sum_{i j} \mathbf{K}_{i j}+\sum_{i j k} \mathbf{K}_{i j k}+\cdots
\end{gathered}
$$

This method has the advantage that techniques used to solve non-relativistic few-body models can be directly applied to relativistic few-body problems. This method has been used extensively in applications; including constituent quark models 11] 12] [13, nuclear reactions [14 15] [16, and electromagnetic probes of hadronic systems [17]

\subsection{Manifestly covariant methods}

These methods involve using equations of quantum field theory that relate different time-ordered Green functions. The simplest of these equations is the Bethe-Salpeter equation. Formally the Bethe-Salpeter Kernel is defined as the difference of the inverse of a product of two-point Green functions and the inverse of a four-point Green function:

$$
K=-\left(G^{-1}\right)_{c}:=G_{0}^{-1}-G^{-1} .
$$

This is equivalent to the Bethe-Salpeter equation for the four point function, $G$,

$$
G=G_{0}+G_{0} K G
$$

In applications $G_{0}$ and $K$ must be modeled. The advantage of these covariant methods is that both spectra and scattering observables can be calculated directly from $G$, without any direct use of the underlying relativistic quantum theory; however the formulas for calculating observables require complete sets of Poincaré irreducible eigenstates between products of fields.

Translational invariance implies the Fourier transforms have the from

$$
\tilde{G}(P)=\tilde{G}_{0}(P)+\tilde{G}_{0}(P) \tilde{K}(P) \tilde{G}(P) .
$$

The existence of a two-body irreducible intermediate states implies that $\tilde{G}(P)$ has a pole a $P^{2}=m^{2}$ :

$$
\tilde{G}(P)=-2 \pi i \frac{\chi(P) \bar{\chi}(P)}{P^{0}-E}+\cdots
$$

where the Bethe-Salpeter wave function $\chi(P)$ is related to the underlying irreducible representation of the Poincaré group by

$$
\chi(P)=\int\left\langle(j, m) \mathbf{P}, \mu\left|T\left(\phi\left(X+\frac{1}{2} x\right) \phi\left(X-\frac{1}{2} x\right)\right)\right| 0\right\rangle \frac{e^{i P \cdot X}}{(2 \pi)^{2}} d^{4} X .
$$


The residue at the pole satisfies the homogeneous Bethe-Salpeter equation

$$
\chi(P)=\tilde{G}_{0}(P) \tilde{K}(P) \chi(P) \quad P^{2}=-M^{2} .
$$

The normalization of the residue is related to the normalization of $G$ by

$$
1=\frac{i}{4 \pi P^{0}} \chi(P) \frac{\partial \tilde{G}^{-1}(P)}{\partial P^{0}} \bar{\chi}(P) .
$$

While the Bethe-Salpeter wave-functions $\bar{\chi}(P)$ do not represent probability amplitudes, given the normalization condition (38) they can be used to compute matrix elements of observables in Poincaré irreducible eigenstates [18].

These methods are most useful in theories like QED where $K$ and $G_{0}$ can be reliably approximated using perturbation theory. Applications of these methods are discussed in the contributions by Bakker [19, Karmanov [20, and Salmé [21] in this volume.

\subsection{Quasipotential methods}

The manifestly covariant methods have the computational disadvantage that the integral equations involve both space and time variables (or four momentum variables). Quasipotential methods reformulate the covariant Bethe-Salpeter equation into an equivalent pair of equations. The first step is to write the product of two-point functions as the sum of a constrained function with the same poles, and a difference,

$$
\tilde{G}_{0}(P)=\tilde{g}_{0}(P)+\tilde{\Delta}(P)
$$

This leads to an equation for a quasipotential, $\tilde{U}(P)$, that replaces the Bethe-Salpeter kernel,

$$
\tilde{U}(P)=\tilde{K}(P)+\tilde{K}(P) \tilde{\Delta}(P) \tilde{U}(P)
$$

and a constrained four-point function that satisfies a quasipotential equation with one less continuous integration variable than the Bethe-Salpeter equation

$$
\tilde{g}(P)=\tilde{g}_{0}(P)+\tilde{g}_{0}(P) \tilde{U}(P) \tilde{g}(P) .
$$

The solution to this equation has the same poles as the Bethe-Salpeter equation. The residues are related to the residues of the Bethe-Salpeter equations

$$
\tilde{K}(P) \chi(P)=\tilde{U}(P) \xi(P)
$$

and they satisfy a normalization condition

$$
1=\frac{i}{4 \pi P^{0}} \xi(P) \frac{\partial g^{-1}(P)}{\partial P^{0}} \bar{\xi}(P) .
$$

In most applications the quasipotental, $\tilde{U}(P)$, is modeled directly, rather than by solving (40) with a model kernel $\tilde{K}(P)$.

The residues can be used in the same way that the $\chi(P)$ are used to calculate observables in the Bethe Salpeter equation.

Example of covariant quasipotential equations are the Gross equation [22] 23].

\subsection{Euclidean methods}

Euclidean relativistic quantum mechanics is a formulation of relativistic quantum mechanics that models the Hilbert space inner product used in the Euclidean formulation 
of quantum field theory by modeling the Euclidean Green functions that appear in the kernel of the Hilbert space scalar product

$$
\langle f \mid g\rangle=\sum_{m n} \int f_{m}^{*}(R x) S_{m+n}(x, y) g_{n}(y) d^{4 m} x d^{4 n} y,
$$

where $S_{m+n}(x, y)$ is an $m+n$ point Euclidean Green function, $R$ is Euclidean time reflection, and the functions $f$ and $g$ are non-zero only when the relative times are positive. The collection of Green functions is called reflection positive if $\langle f \mid f\rangle \geq 0$. Poincaré generators are constructed from Euclidean generators as follows; Euclidean generators that anticommute with $R$ are multiplied by $i$ and generators that commute with $R$ remain unchanged. The resulting operators are Hermitian with respect to the inner product (44) and satisfy the Poincaré commutation relations. The dynamics can be expressed in terms of $e^{-\beta H}$, which is represented by a translation of all Euclidean times by $\beta>0$. This operator can be used like the Hamiltonian to compute bound and scattering observables.

This method is closely related to Euclidean version of the Schwinger-Dyson equations. The advantage of the quantum mechanical interpretation is that all calculations can be performed in the Euclidean framework without analytic continuation. Model calculations have been used to demonstrate that $e^{-\beta H}$ can be used to calculate GeVscale scattering observables [24] using the relation

$$
\left\langle\psi\left|\Omega_{+}^{\dagger} \Omega_{-}\right| \phi\right\rangle \quad \Omega_{ \pm}=\lim _{n \rightarrow \pm \infty} e^{-i n e^{-\beta H}} \Pi e^{+i n e^{-\beta H_{0}}}
$$

where $\Pi$ is a mapping from an asymptotic channel space to the Hilbert space.

\section{Summary}

In this contribution I demonstrated the unifying role played by irreducible representations of the Poincaré group in essentially all formulations of relativistic few-body quantum theory. These representations arise as a result of the requirement that all quantum mechanical observables have the same value in all inertial coordinate systems, which requires that the dynamics is given by a unitary representation of the Poinaré group. The irreducible representation are the elementary building blocks of these dynamical representations.

The author would like to acknowledge and thank numerous collaborators who have significantly contributed the physics discussed in these proceedings. This includes Fritz Coester, Charlotte Elster, Walter Glöckle, Jacek Golak, Hirouyki Kamada, Brad Keister, Phil Kopp, Ting Lin, Roman Skibínski, Henryk Witała and Yunfei Huang.

\section{References}

[1] E. P. Wigner (1939), Ann. Math. 40,140.

[2] P. A. M. Dirac (1949), Rev. Mod. Phys. 21,392.

[3] S. Weinberg (1995), The Quantum Theory of Fields, Vol 1, Cambridge University Press, Ch. 4.

[4] S. N. Sokolov (1977), Dokl. Akad, Nauk SSSR, 233, 575.

[5] B. Keister and W. N. Polyzou (2012), Phys. Rev. C 86,014002.

[6] F. Coester and W. N. Polyzou (1982), Phys. Rev. D 26,1348. 
[7] H. W. Crater, R. Becker, C. Y. Wong, and P. Van Alstine (1992), Phys. Rev. D $46,5117$.

[8] H. W. Crater and C.-Y. Wong (2012), Phys. Rev., D 85, 116005.

[9] H. W. Crater and Peter Van Alstine, (1988) Phys. Rev. D 37, 1982.

[10] H. W. Crater, James Schiermeyer, (2010) Phys. Rev. D 82,094020.

[11] R.F. Wagenbrunn, S. Boffi, W. Klink, W. Plessas, and M. Radici (2001), Phys. Lett. B511, 33.

[12] Wolfgang Schweiger (these proceedings)

[13] S. Capstik and B. D. Keister Capstik, Phys.Rev. D 513598.

[14] T. Lin, Ch. Elster, W. N. Polyzou, W. Glöckle (2008), Physics Letters, B660, 345 .

[15] H. Witała, J. Golak, R. Skibínski, W. Glöckle, H. Kamada, and W. N. Polyzou, (2011), Phys. Rev. C 83, 044001.

[16] Emanuele Pace, Giovanni Salme, Sergio Scopetta, Alessio Del Dotto, Matteo Rinaldi (2013), Few Body Syst. 54, 1079-1082.

[17] V.A. Karmanov, A.V. Smirnov, (1994) Nuc. Phys., A575, 520-548

[18] K. Huang and A. Weldon (1975), Phys. Rev. D 11,257.

[19] B. Bakker (these proceedings).

[20] V.A. Karmanov, J. Carbonell (these proceedings).

[21] G. Salmé (these proceedings).

[22] Franz Gross and Alfred Stadler, (2008), Phys. Rev. C 78, 014005.

[23] Elmar P. Biernat, Franz Gross, Teresa Pena, Alfred Stadler (these proceedings).

[24] P. Kopp and W. N. Polyzou (2012), Phys. Rev. D 85,016004. 